Research Article

\title{
Prediction of Perceived Utility of Consumer Online Reviews Based on LSTM Neural Network
}

\author{
Hu Wang, Tianbao Liang, and Yanxia Cheng $\mathbb{D}$ \\ School of Management, Wuhan University of Technology, Wuhan 430070, Hubei, China \\ Correspondence should be addressed to Yanxia Cheng; chengyanxia221@whut.edu.cn
}

Received 18 May 2021; Revised 3 June 2021; Accepted 22 June 2021; Published 2 July 2021

Academic Editor: Sang-Bing Tsai

Copyright (C $2021 \mathrm{Hu}$ Wang et al. This is an open access article distributed under the Creative Commons Attribution License, which permits unrestricted use, distribution, and reproduction in any medium, provided the original work is properly cited.

\begin{abstract}
Perceived value is the customer's subjective understanding of the value they obtain and is their subjective evaluation of the product or service they enjoy. This value is deducted from the cost of the product or service. In order to understand and predict the specific cognition of consumers on the value of products or services and distinguish it from the objective value of products or services in the general sense, this paper uses the in-depth learning method based on LSTM to establish a model to predict the perceived benefits of consumers. It is a challenging task to analyze the emotion of consumers or recognize the perceived value of consumers from various texts of online trading platforms. This paper proposes a new short-text representation method based on bidirectional LSTM. This method is very effective for forecasting research. In addition, we also use the attention mechanism to learn the specific emotional vocabulary. Short-text representation can be used for emotion classification and emotion intensity prediction. This paper evaluates the proposed classification model and regression data set. Compared with the baseline of the corresponding data set, the contrast of the results was $93 \%$. The research shows that using deep neural network to predict the perceived utility of consumer comments can reduce the intervention of artificial features and labor costs and help predict the perceived utility of products to consumers.
\end{abstract}

\section{Introduction}

Online shopping is a brand new shopping experience, which is a kind of e-commerce. Consumers use their mobile terminal devices to enter the online shopping platform to buy the goods and services they want. It allows consumers to visit the official website of mobile online retailers through a direct browser or search among other suppliers through an engine that directly uses mobile shopping websites. It is used to let consumers find services or products they are interested in. Online consumption has become one of the mainstream consumption methods, and online shopping users spend more and more money on online shopping [1-3]. This also makes the customer's satisfaction with the product, and good evaluation of the online store is very important. It is very important to study and identify the perceived value of the target customers and find out the product value field that customers are most concerned about in the contemporary society because the customer's perceived value plays an important role in shaping the connotation of the product and improving the quality of the product [4]. The research on the prediction of consumer's online appraisal and perceived utility can enable stores to provide help in the application of products and services.

In natural language processing, sensory classification refers to marking a text as a whole that produces positive or negative emotions. On the other hand, the task of emotion perception is to associate words, phrases, or documents with a set of predefined emotions from mental patterns such as fear, joy, anger, and sadness. The intensity of emotional expression in text helps quantify and compare subjective expression and also helps identify the perceived value. In the recent years, Chinese scholars have proposed several indepth learning models for these tasks [5-7]. This article discusses some important initiatives, most of which use a "word"-based approach. Our observation shows that the text generated by consumers in online comments usually uses innovative expressions, uses new or insufficient vocabulary, and combines icons, numbers, and symbols to express them. 
Bartoli et al. pointed out that consumer reviews are important references for e-commerce companies to collect resource information and make correct decisions. They evaluated the possibility of customers being deceived by automatically generating hundreds of false reviews on restaurants, and the results confirmed that customers are largely affected by such false reviews [8]. On the other hand, Qian et al. has established an identification pattern based on LSTM to determine the intention of consumer travel. The intention of travel consumption in chat robots refers to the willingness of users to purchase products or services in order to meet their travel needs. The author suggests that the intention of consumer products should be determined to enhance user experience. The traditional method of consumer intention recognition is mainly based on template matching or artificial feature sets, which is time-consuming, laborious, and difficult to expand. The author takes the task of consumer intention recognition as a classification problem and combines the deep learning method to identify. This method does not need to construct feature sets or matching templates manually. Specifically, the author uses the convolutional long short-term memory (LSTM) neural network model to identify travel consumption intention. First, the convolution neural network (CNN) is created to extract the features of user chat texts, and then the feature combination. Then, the feature is sent to LSTM to study the feature of feature representation and output the classification result. The experimental results show that the convolution LSTM model is $2 \%$ better than the best baseline method in F-measure [9]. But there are errors in the data.

Word-based systems cannot effectively learn these expressions, which affects the performance of consumer intention recognition or perceived value classification. In this paper, we propose an effective method to detect and classify emotion intensity, which does not depend on three or five scales of fixed words. In addition, the innovation of this paper is to propose the use of the attention mechanism to improve the efficiency of the learning system, which proves that the proposed combination is superior to the state-ofthe-art perceptual utility classification mechanism.

\section{Proposed Method}

\subsection{LSTM Neural Network}

2.1.1. Concept of LSTM. A recurrent neural network is a kind of artificial neural network. It has memory properties, including long-term memory and short-term memory. It is a deep learning algorithm. The long short-term memory network has a feedback connection, which can process a single data point as well as the entire data sequence [10]. A general LSTM unit consists of a unit, an input gate, an output gate, and a forgetting gate. The unit remembers the value in any time interval, and three doors adjust the information flow in and out of the unit. LSTM is developed for the problem of explosion and vanishing gradient that may be encountered in traditional RNN training. Compared with RNNs, HMM, and other sequential learning methods, LSTM can adapt to learning with certain interval, which is an advantage [11, 12].
2.1.2. Development and Structure of LSTM. A long shortterm memory network is a common cyclic neural network; LSTM networks were proposed by Liu et al. and were improved and extended by many people in the subsequent work $[13,14]$. They are very effective in many problems, and nowadays they are widely used. A general LSTM architecture consists of one unit (the storage part of the LSTM unit) and three "regulators" (usually called gates), which are the regulators of information flow inside the LSTM unit: input gate, output gate, and forgetting gate. Some variants of the LSTM unit do not have one or more of these doors, or there may be other doors. For example, a gated recursive unit (Gru) does not have an output gate. Intuitively, cells are responsible for tracking the dependencies between elements in the input sequence. The input gate controls the range of new values flowing into the cell, forgetting the range of the gate control value remaining in the cell. The value in the output gate control cell is used to calculate the range of output activation of the LSTM cell $[15,16]$.

2.1.3. Application of LSTM. LSTM networks are a form of RNN, which can effectively generate character sequences with long-distance structure. Based on the character-level LSTM rrn machine translation task, this paper proves its superiority. The RNN is used for Q \& A, and the NLG system produces correct answers to questions expressed in natural language. This work provides a dialogue system that, as part of the dialogue, can produce a smoother response [17]. The LSTM framework is shown in Figure 1. A significant application of LSTM in NLG is to generate descriptive captions for image description and video sequences. In this way, there is data collection for the online evaluation of consumers later.

Motion recognition based on the position of the main joints of the human body has attracted wide attention in three-dimensional space; we have designed a deep network model for human behavior recognition. The model has high efficiency and accuracy in capturing motion, spatial characteristics, and time dependence. For any machine learning project, choosing the right algorithm is a time-consuming and laborious process. Without increasing the complexity of the model, the nonstationary problem of long-term dynamics can be solved more effectively. At the same time, a variety of modes, RGB images, and optical flow are used to train the control door so as to better control the information entering/leaving the storage unit [18].

Semantic analysis is the process of extraction and interpretation from natural language and the basis of natural language processing. In the past two decades, a lot of work has proposed solutions for virtual or real autonomous agents. All methods, from the initial grammar method to the pure statistics method and the hybrid method, follow the grammar theory. At present, the most effective model promotes the dependence on the established language theory to represent the action semantics in user commands. A statistical semantic analyzer based on support vector machine is trained on the human robot interaction corpus. It is an attempt to build a bridge between NLU of robots and voice representation theory with more linguistic and cognitive significance [19]. 


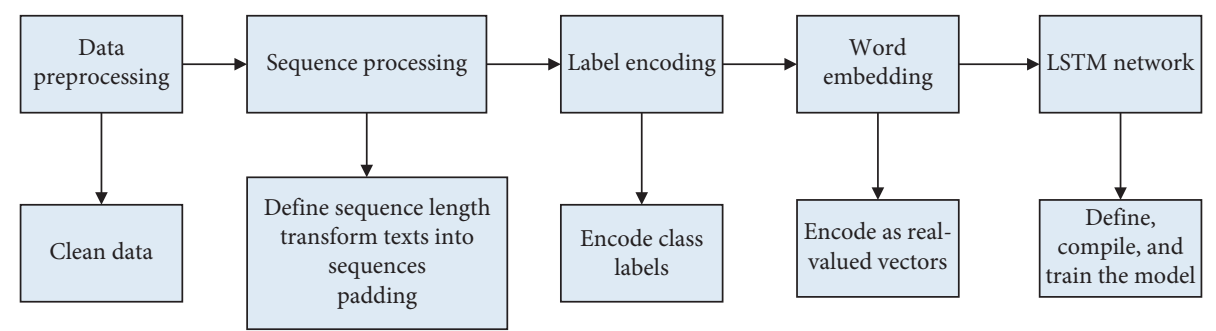

Figure 1: Processes of the LSTM model.

\subsection{Perceived Utility of Consumers}

(1) For the same product, customers tend to be more inclined to buy from companies that they think can provide the highest perceived value. Customer perceived value (CPV) refers to the difference between potential customers' evaluation of all benefits and all costs of products and perceived substitutes [20, 21]. The total customer cost includes the buyer's time, energy, and psychological cost [22]. The buyer evaluates these elements together with the monetary cost to form the total customer cost. The buyer will purchase from any source that he believes provides the highest perceived customer value. This decision theory can help the seller to sell the product to the buyer successfully.

2.3. Predict Consumer Behavior. On the basis of consumer investigation and research, the prediction of consumer's purchase behavior analyzes the consumer's consumption ability, consumption level, and consumption structure; reveals the consumption characteristics and demand differences of different consumer groups; judges the changes of consumer's purchase habits, consumption tendency, and consumption preference; and studies what, how much, when, and where consumers purchase-purchase behavior and its changes, such as purchase, who to buy, how to buy, etc. The purpose of consumer purchase behavior prediction is to provide basis for market potential measurement, target market selection, product development, and marketing strategy formulation [23-25].

With the development of e-commerce, more and more scholars have paid attention to the information search behavior of consumers, among which the impact of online comments on consumers is the focus of scholars' attention. Many scholars at home and abroad have studied online reviews $[26,27]$, and the research of predictive models has also become a research hotspot.

\section{Experiments}

3.1. Data Preprocessing. The text on consumer online reviews has a high degree of variability in grammatical structure and syntax. As mentioned earlier, online consumption platforms limit the limited characters to one comment. This restriction does not include the user name of the user. In addition, if the expression is encoded in UTF format, it is treated as a single character. In addition to text, consumer reviews also include some pictures and videos, which are all effective information for making customer perceived value judgments. Before analyzing the perceived value, we need to perform feature processing on this information and remove unnecessary interference elements to reduce noise. For the word level, the following cleanup is applied. Application cleaning is to make the data characteristics of the predictive model distinct:

(1) Tags are important to determine emotions or users' intentions. Users often refer to popular tags to show their intentions. Delete the “ $\square$ " symbol and retain the word itself.

(2) The user name refers to words beginning with “@,” which usually does not provide emotional information. As a result, such terms are completely removed from tweets. However, if the text contains multiple comments as part of a single conversation, what the user mentions will be an important aspect.

(3) Link hyperlinks are removed because they do not convey emotional information in the text.

(4) Delete emoticons (e.g., " $(\geq \omega \leq) /{ }^{\prime \prime}\left({ }^{\prime}\right.$ m) $)$ ", etc.).

(5) Remove extra space.

(6) For character-level models, the user mentions and removes the label symbol, as described earlier, while the emoticon is replaced with the corresponding description of the emoticon; for example, $U+1 \mathrm{f} 600$ is replaced with the word "smiling face." This modification is helpful to capture the emotion described by this emoticon in the form of text.

Data sets that can be used for sentiment analysis are collected from a large number of comments and annotated by a large number of users. Because perception itself is a subjective problem, users may have different emotional perceptions of the same text. In addition, users' internal beliefs or knowledge also plays a role in emotional perception. Table 1 shows sample comments from the data set to illustrate these. Class represents the perceived utility of the consumer; -2 represents the most negative, i.e., the consumer feels very unworthy of purchase; 2 represents the most positive, i.e., the consumer feels very worthy of purchase; and 0 represents neutral.

It is difficult to capture and observe the dependence of comments from the pretrained word embedding. With the increase of perceptual prediction granularity, the 
TABle 1: Sample comments for the data set.

\begin{tabular}{lc}
\hline Examples of consumer's reviews & Class \\
\hline Fantastic, wonderful, worth buying & 2 \\
Just so so, not bad & 1 \\
Not required, unnecessary & 0 \\
Not recommended, not good & -1 \\
Very bad, do not buy again & -2 \\
\hline
\end{tabular}

noise becomes more obvious. For this kind of task, it is impossible to train your own embedding because there are about hundreds of annotated data, which are very low for learning embedding. Character-level embedding is recommended to handle these types of dependencies. Character-level embedding is necessary to create such a representation of words outside the vocabulary. In addition to character-level embedding, emotional dependence on tags can be modeled by attention mechanism, which is very popular in the field of computer vision. Recursive networks are effective in processing time data. Most commonly, the recurrent neural network (RNN) is trained by random gradient descent (SGD), and the gradient of training criteria is calculated by time backpropagation algorithm. The long short-term memory network (LSTM) is a special case of neural network, which solves the classic problem of gradient disappearance (or explosion). The LSTM architecture used in this paper is the same as that proposed in [27] and is controlled by the following equation:

$$
\begin{aligned}
& i_{t}=\tanh \left(W_{x i} x_{t}+W_{a i} a_{t-1}+b_{i}\right), \\
& j_{t}=\operatorname{sigm}\left(W_{x j} x_{t}+W_{a j} a_{t-1}+b j\right), \\
& k_{t}=\operatorname{sigm}\left(W_{x k} x_{t}+W_{a k} a_{t-1}+b_{k}\right), \\
& o_{t}=\tanh \left(W_{x o} x_{t}+W_{a o} a_{t-1}+b_{o}\right), \\
& c_{t}=c_{t-1} \oplus k_{t}+i_{t} \oplus j_{t}, \\
& h_{t}=\tanh \left(c_{t}\right) \oplus k_{t} .
\end{aligned}
$$

In the above equation, $w^{*}$ variable is the weight matrix and $B^{*}$ variable is the deviation. Operation $\oplus$ represents the vector product of elements. The variable CT represents the memory of the LSTM in time step $t$. HT is called hidden state.

The two-way recurrent neural network (RNN) was originally used for speech recognition. Gradually, they are applied to different tasks, such as syntactic analysis and oral comprehension. A BiLSTM network is a brnns using the LSTM hidden layer. BiLSTM is a stack of two LSTMs: forward processing information from $t=1-T$, while reverse processing information from $t=T-1$. The equations of the LSTM layer remain unchanged, and the random gradient can be used for training. And then achieve the data processing of the evaluation label.

3.2. Attention Mechanism. Attention mechanism has recently been proposed to model long-term dependency. It allows the model to have a more direct relationship between states at different points in the time domain. According to the definition, the model that generates the hidden state HT in each time step is given. Based on the definition, a model that generates the hidden state HT in each time step is given. According to equation (2) based on the attention model, the vector $C$ is calculated as the weighted average value of the state sequence $H$.

$$
c_{t}=\sum_{i=1}^{T} a_{t i} h_{i}
$$

It is defined as the total number of time steps in the sequence, which is the weight vector calculated at each time step of each hidden state sequence $s$ and depends on the model output and input. The weight of input and output values is obtained from

$$
\begin{aligned}
e_{w i} & =a\left(s_{t-1}, h_{i}\right), \\
a_{t i} & =\frac{\exp \left(e_{t i}\right)}{\sum_{k=1}^{T} \exp \left(e_{t k}\right)} .
\end{aligned}
$$

Given a value and a previous state, there is a function of scalar importance value that can be considered to be computed.

\section{Discussion}

The model is evaluated on a large data set, Tianchi data set. This data set represents the emotion of online consumption platforms. The Tianchi data set is used for classification and regression tasks. These data sets are annotated using crowdsourcing.

4.1. Classification Task of the Data Set. This data set provides online consumer platform reviews and their corresponding reviews. Classification is the most negative, negative, neutral, positive, and positive class in the range of -2 to 2 . Sample comments for this data set are shown in Table 1. Figure 2 shows the histogram of comment classification, with a relatively small proportion of neutral attitudes, and it can be seen from the histogram that most comments have strong emotions. The data present clearer results, which are very clear about whether they are worth or not.

4.2. Model Prediction Accuracy. During the experiment, 6420 comments in the data set were sorted by category labels and then predicted and classified on the BiLSTM model and the traditional LSTM, respectively. The classification accuracy of the model on the training set and test set after each iteration is recorded, and the recorded results are shown in Figure 3. It can be seen from the figure that the accuracy rate of classification increases with iteration. In the process of 50 iterations, the accuracy rate of the BiLSTM model in training data reaches $93.13 \%$ at most, and the accuracy rate in LSTM reaches $82.84 \%$ at most. This can more accurately predict the perceived utility of consumers' online reviews. 


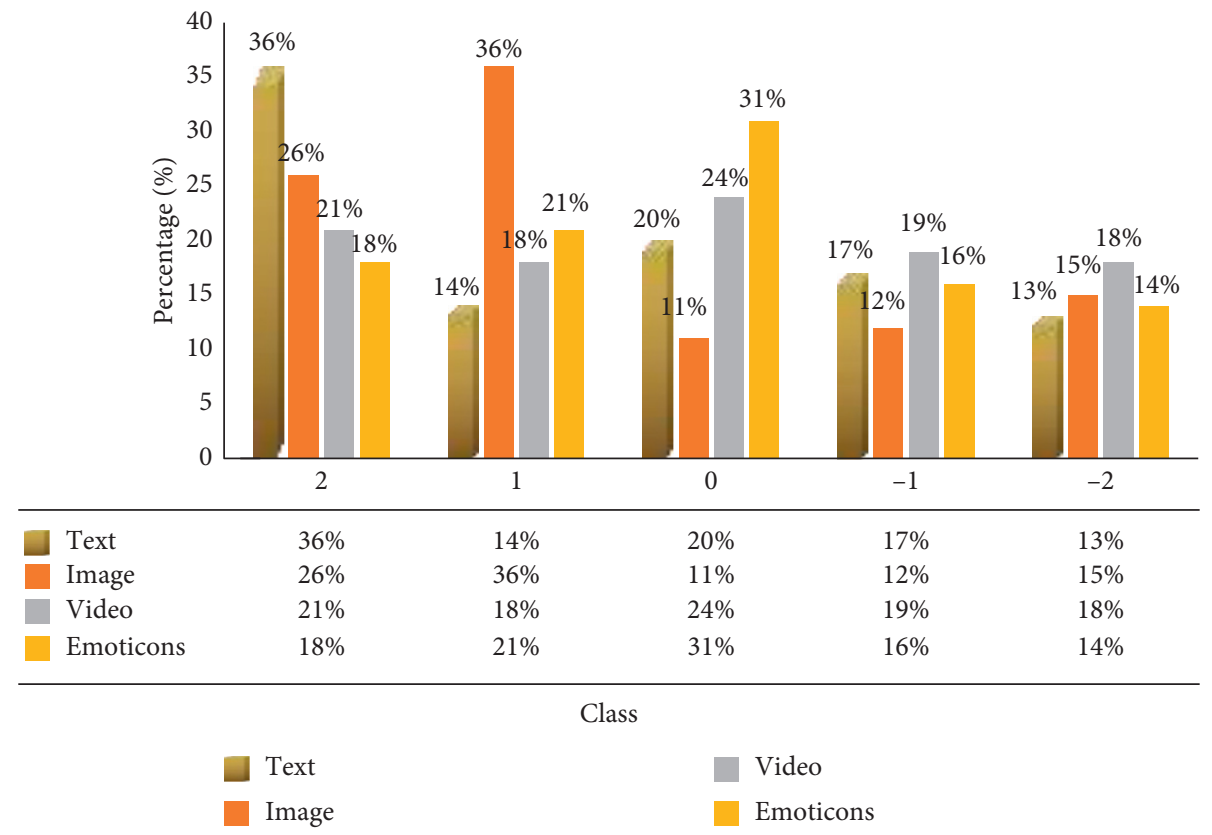

Figure 2: The distribution of consumer reviews.

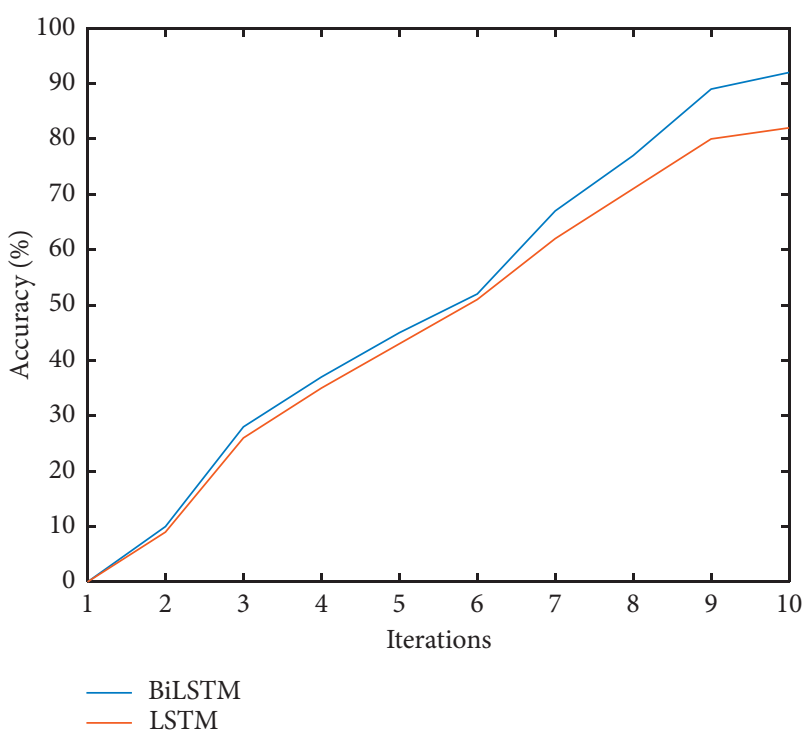

FIGURE 3: The accuracy comparison of different iterations between BiLSTM and LSTM.

4.3. Loss Function. During the experiment, the 6420 tags in the data set were sorted by category tags, and then one comment body action was randomly selected from every 15 adjacent actions to be put into the test set, and other human actions were put into the training set. In this way, the class distribution of training set and test set is consistent. Then, the training model is trained 50 times with all the actions of the training set, and the data of the training set will be randomly rearranged in each iteration. For this reason, the loss function value of the model after each iteration and the classification accuracy of the model on the training set and test set are recorded, and the recorded results are shown in Figure 4 . It can be seen from the figure that the loss function

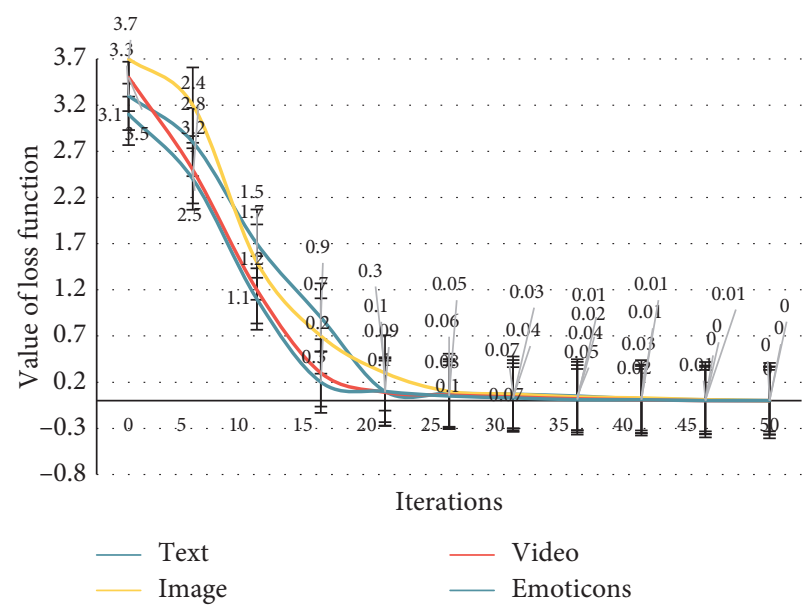

Figure 4: The value of loss function with the iterations.

of the classification decreases with the number of iterations, and the final changes will tend to be stable at the level of 0.03 , which is also in line with the general training process of the neural network model.

4.4. Character Frequency of Consumer Comments. To predict the perceived utility of consumers, we can not only use classification method to identify but also refer to the consideration of consumers on satisfied products. We can summarize the aspects that businesses should pay attention to products, try to bring the maximum perceived value to customers, and promote a win-win virtuous cycle in which customers' satisfaction is higher and higher and businesses' products are more and more worthy. As shown in Figure 5, among the comments with high perceived utility, the comments with good quality are the most, followed by the 


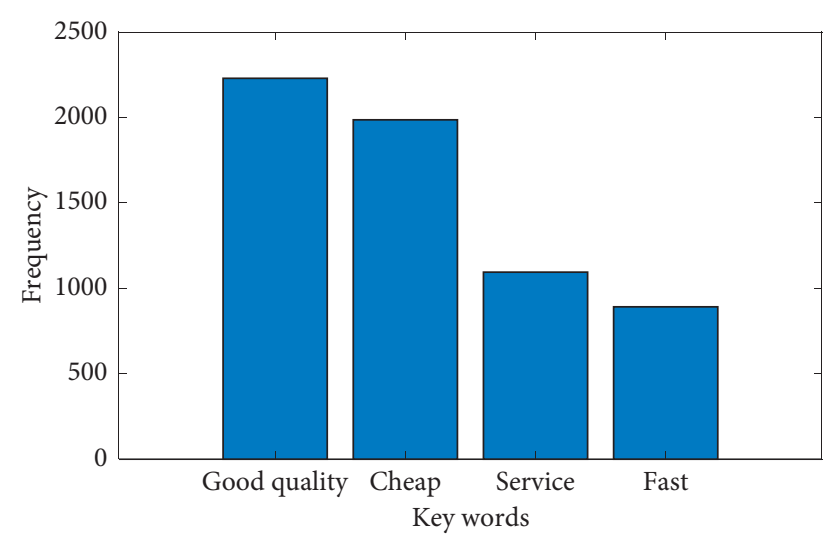

FIgURE 5: The frequency of keywords in good reviews.

comments with low price, good service, and fast delivery. Thus, if we want to improve the perceived utility of customers, these four aspects are the best places for businesses to improve.

Based on the perceived value characteristics of consumers' online shopping, enterprises can adopt the following strategies when formulating online marketing strategies to adapt to the perceived psychology of different types of consumers:

(1) Strengthen the management of goods purchase channels, ensure the quality of online goods, enable consumers to buy high-quality and low-cost goods online, strengthen the service awareness of enterprises, improve the level of presale and after-sale services, and improve the perceived effectiveness of customers on goods.

(2) Based on the consumption ability of online consumers, with customers as the core, reasonable commodity prices are formulated. By providing commodities with different price levels to meet the needs of customers at different consumption levels, the tangible cost and psychological risk cost of online shopping are reduced.

(3) For products sold online, quality is the first element. In addition to quality, we should diversify the products in terms of style and color to meet the needs of different consumers, so as to reduce the cost of consumer online shopping information collection.

(4) By optimizing service, doing business in good faith, establishing corporate image, reducing the perceived risk of customers' online shopping, reducing the risk cost, and creating a good atmosphere for customers' online shopping.

Because the factors that affect the perceived utility of Internet consumers include not only cost but also consumer preferences and Internet services, we need to further expand the independent variables that affect the perceived utility of Internet consumers, and further improve the perceived utility model of Internet consumers, so as to fully reveal the characteristics of consumer decision-making behavior in the Internet environment.

\section{Conclusions}

In this experiment, BiLSTM is used to train, develop, and test the data set of consumer reviews, and an improved BiLSTM recurrent neural network system is proposed to better control how much information each memory unit outputs. The forgetting gate and input gate are combined into a single update gate. The BiLSTM network is a brnns using the LSTM hidden layer. BiLSTM is a stack of two LSTMs: forward processing information from $t=1-T$, while reverse processing information from $t=T-1$. The equations of the LSTM layer remain unchanged, and the random gradient can be used for training. In this way, the improved BiLSTM model can learn long-term historical information and has stronger robustness. Based on the improved method, experiments are carried out on the Tianchi data set. Based on the improved BiLSTM recurrent neural network system, the recognition rate is effectively improved to $93 \%$, the perceived value of consumers is successfully predicted, and the robustness of online comment recognition system is improved.

In this paper, deep learning is applied to demand side LSTM, and a network neural-based LSTM framework is proposed. Specifically, the problem of training with customer load data is studied carefully. If considering the scale of neural network, the amount of available single load data is relatively small, overfitting may occur during training. The experimental results show that LSTM can be trained well by using the user's three-year load data. The results can be further extended to several aspects. For example, it is expected to train LSTM better when big data are available and further explore a better LSTM structure. For example, convolutional neural network is used as a time series prediction model. In addition, analyzing the exact conditions of LSTM (such as load type, load mode, and date) is helpful to select the appropriate prediction model for an individual. This provides a certain reference value for the establishment of predictive models in various fields in the future.

This paper proposes a robust short-text representation method, which can effectively predict and classify five-line emotion. The proposed model is evaluated on two data sets, and the results show significant improvement without considering any hand-made characteristics. This work can be extended to more data sets. If transmission learning is combined on different data sets, its robustness will be better. In this work, the main focus is on the automatic classification of positive and negative reviews on online consumer platforms. Generally, the problem of machine learning classification requires predefined characters. The development of neural networks is to optimize the model and maximize accuracy. Although there are problems with an insufficient in-depth understanding of the research of neural networks in this article, neural networks are still used to solve many predictive modeling problems for sequence classification. 


\section{Data Availability}

No data were used to support this study.

\section{Conflicts of Interest}

The authors declare that there are no conflicts of interest.

\section{Acknowledgments}

This work was supported by the National Social Science Foundation of China (Grant no. 16BGL089).

\section{References}

[1] M. Zhang, D. Wu, and R. Xue, "Hourly prediction of PM 2.5 concentration in Beijing based on Bi-LSTM neural network," Multimedia Tools and Applications, vol. 2, no. 8, pp. 1-14, 2021.

[2] E. Ayvaz, K. Kaplan, and M. Kuncan, "An integrated LSTM neural networks approach to sustainable balanced scorecardbased early warning system," IEEE Access, vol. 8, pp. 37958-37966, 2020.

[3] A. Abdelnasser, M. Z. Rashad, and S. E. Hussein, "A two-layer water demand prediction system in urban areas based on micro-services and LSTM neural networks," IEEE Access, vol. 8, pp. 147647-147661, 2020.

[4] R. Chakraborty, J. Lee, S. Bagchi-Sen, S. Upadhyaya, and H. Raghav Rao, "Online shopping intention in the context of data breach in online retail stores: an examination of older and younger adults," Decision Support Systems, vol. 83, pp. 47-56, 2016.

[5] Y. Chen, X. Yan, W. Fan, and M. Gordon, "The joint moderating role of trust propensity and gender on consumers' online shopping behavior," Computers in Human Behavior, vol. 43, pp. 272-283, 2015.

[6] E. Wong and W. Yan, "Customer online shopping experience data analytics: integrated customer segmentation and customised services prediction model," International Journal of Retail \& Distribution Management, vol. 46, no. 3, pp. 10-22, 2018.

[7] O. E. Omar and A. Hirst, "Apparel shopping: a focus on the attitudes of women towards online shopping," International Journal of Electronic Marketing \& Retailing, vol. 1, no. 2, pp. 169-182, 2017.

[8] A. Bartoli, A. D. Lorenzo, and E. Medvet, "Best dinner ever!!!”: automatic generation of restaurant reviews with LSTM-RNN [C].2016," in Proceedings of the IEEE/WIC/ACM International Conference on Web Intelligence, vol. 10, no. 12, pp. 1-4, Omaha, NE, USA, 2017.

[9] Y. Qian, X. Ding, and T. Liu, "Identification method of user's travel consumption intention in chatting robot," Scientia Sinica (Informationis), vol. 391, no. 1, pp. 83-84, 2017.

[10] K. Greff, R. K. Srivastava, J. Koutnik, B. R. Steunebrink, and J. Schmidhuber, "A search space odyssey," IEEE Transactions on Neural Networks \& Learning Systems, vol. 28, no. 10, pp. 2222-2232, 2015.

[11] K. Chen and Q. Huo, "Training deep bidirectional LSTM acoustic model for LVCSR by a context-sensitive-chunk BPTT approach," IEEE/ACM Transactions on Audio, Speech, and Language Processing, vol. 24, no. 7, pp. 1185-1193, 2017.

[12] J. Liu, G. Wang, and L.-Y. Duan, "Skeleton-based human action recognition with global context-aware attention LSTM networks," IEEE Transactions on Image Processing, vol. 27, no. 4, pp. 1586-1599, 2017.

[13] Y. Liu, D. Shuting, M. Lu et al., "LSTM based reserve prediction for bank outlets," Tsinghua Science and Technology International Journal on Information Science, vol. 24, no. 1, pp. 77-85, 2019.

[14] J. Yan, Y. Qi, and Q. Rao, "LSTM-based with deterministic negative sampling for API suggestion," International Journal of Software Engineering and Knowledge Engineering, vol. 29, no. 7, pp. 1029-1051, 2019.

[15] D. Park, S. Kim, Y. An, and J.-Y. Jung, "LiReD: a light-weight real-time fault detection system for edge computing using LSTM recurrent neural networks," Sensors, vol. 18, no. 7, pp. 2110-2113, 2018.

[16] B. Meng, X.J. Liu, and X. Wang, "Human action recognition based on quaternion spatial-temporal convolutional neural network and LSTM in RGB videos," Multimedia Tools \& Applications, vol. 38, no. 11, pp. 26901-26918, 2018.

[17] R. Zazo, P. Sankar Nidadavolu, N. Chen, J. GonzalezRodriguez, and N. Dehak, "Age estimation in short speech utterances based on LSTM recurrent neural networks," IEEE Access, vol. 6, no. 1, pp. 22524-22530, 2018.

[18] M. Coto-Jiménez, J. C. Goddard, LSTM deep neural networks post filtering for enhancing synthetic voices," International Journal of Pattern Recognition \& Artificial Intelligence, vol. 32, no. 1, pp. 57-65, 2017.

[19] F. Liu, X. Zhao, P. Y. K. Chau, and Q. Tang, "Roles of perceived value and individual differences in the acceptance of mobile coupon applications," Internet Research, vol. 25, no. 3, pp. 471-495, 2015.

[20] G. Naganathan, K. Kuluski, A. Gill, L. Jaakkimainen, R. Upshur, and W. P. Wodchis, "Perceived value of support for older adults coping with multi-morbidity: patient, informal care-giver and family physician perspectives," Ageing and Society, vol. 36, no. 9, pp. 1891-1914, 2015.

[21] Y. Liu, J.-W. Bi, and Z.-P. Fan, "Ranking products through online reviews: a method based on sentiment analysis technique and intuitionistic fuzzy set theory," Information Fusion, vol. 36, pp. 149-161, 2017.

[22] J. Jin, P. Ji, and R. Gu, "Identifying comparative customer requirements from product online reviews for competitor analysis," Engineering Applications of Artificial Intelligence, vol. 49, pp. 61-73, 2016.

[23] N. Sahoo, C. Dellarocas, and S. Srinivasan, "The impact of online product reviews on product returns," Social Science Electronic Publishing, vol. 29, no. 3, pp. 1-12, 2018.

[24] A. Y. L. Chong, B. Li, W. Eric, T. Ngai, and F. Lee, "Predicting online product sales via online reviews, sentiments, and promotion strategies," International Journal of Operations \& Production Management, vol. 36, no. 4, pp. 358-383, 2016.

[25] C. M. Reich and S. M. Hack, E. A. Klingaman, Consumer satisfaction with antipsychotic medication-monitoring appointments: the role of consumer-prescriber communication patterns," International Journal of Psychiatry in Clinical Practice, vol. 22, no. 2, pp. 1-6, 2017.

[26] S. P. F. Bonny, J.-F. Hocquette, and D. W. Pethick, "Untrained consumer assessment of the eating quality of beef: 1. a single composite score can predict beef quality grades," Animal, vol. 11 , no. 8, pp. 1-10, 2016.

[27] A. Marakhimov and J. Joo, "Consumer adaptation and infusion of wearable devices for healthcare," Computers in Human Behavior, vol. 76, no. 11, pp. 135-148, 2017. 